Pacific Journal of Mathematic 


\title{
STRUCTURE SPACES FOR SANDWICH SEMIGROUPS
}

\author{
K. D. Magill, JR., P. R. Misra and U. B. Tewari
}

For a given semigroup, a structure space is associated in a natural way with each left ideal of that semigroup. In this paper, we determine this space when the semigroup is a sandwich semigroup satisfying appropriate conditions and the left ideal is the kernal of the semigroup.

Most of the attention in [5] and in subsequent papers [6], [7] was focused on the case where the left ideal is the kernel of the semigroup in which case the term $\mathscr{r}$-structure space was used. We recall briefly the definition. Let $S$ be any semigroup with kernel $K$. The requirement that $S$ have a kernel (i.e., smallest two-sided ideal) is, of course, a restriction since the intersection of all two-sided ideals of a semigroup may well be empty. A bond on $S$ is any subset $A$ of $S \times K$ such that for each finite subset $\left\{\left(s_{i}, k_{i}\right)\right\}_{i=1}^{n}$ of $A$, the set of equations $\left\{s_{i} x=k_{i}\right\}_{i=1}^{n}$ has a common solution $x$ in $K$. Maximal bonds are called ultrabonds and the collection of all ultrabonds on $S$ is denoted by $\mathscr{U}(S)$. For each $(s, k) \in S \times K$, define

$$
H(s, k)=\{A \in \mathscr{C}(S):(s, k) \in A\}
$$

and topologize $\mathscr{U}(S)$ by taking all sets of the form $H(s, k)$ as a subbasis for the closed subsets. $\mathscr{U}(S)$, with this topology, is the $\mathscr{K}$-structure space of $S$. It was shown in [5] and [6] that for many topological spaces $X$, the $\mathscr{K}$-structure space of $S(X)$, the semigroup of all continuous selfmaps of $X$ turns out to be $\beta X$, the Stone-Čech compactification of $X$.

In [5] a number of results were proved about the $\mathscr{K}$-structure space of an abstract semigroup with certain conditions being placed on that semigroup. One of the conditions was that the semigroup contain a left identity. This didn't seem to be much of a restriction at the time since we were primarily interested in applying these results to $S(X)$ which has a two-sided identity. But once we became more interested in sandwich semigroups, the requirement that the semigroup contain a left identity became a serious obstacle. Let us recall the definition of a sandwich semigroup $S(X, Y, \alpha)$. $X$ and $Y$ are topological spaces and $\alpha$ is a continuous function from $Y$ into $X . S(X, Y, \alpha)$ is the semigroup of all continuous functions from $X$ into $Y$ where the product $f g$ of two elements $f$ and $g$ in $S(X, Y, \alpha)$ is defined by $f g=f \circ \alpha \circ g$. The semigroup $S(X, Y, \alpha)$ is referred to as a sandwich semigroup with sandwich function $\alpha$. 
The problem is that $S(X, Y, \alpha)$ contains a left identity if and only if $\alpha$ maps $Y$ homeomorphically onto a retract of $X$ and this is far too restrictive for our purposes here. However, Anne Hudson (private communication) and H. O. Kim [2] observed independently that the requirement that the semigroup contain a left identity is not really necessary, at least, not in order to get a number of the results we obtained in [5]. So this meant that it would not be unreasonable to try to use these results (or more accurately, slightly amended versions of these results) to determine just what the structure space of $S(X, Y, \alpha)$ is. This problem is the main thrust of this paper and we show that for certain spaces $X$ and $Y$, if $\alpha$ is any closed function from $Y$ into $X$ satisfying some rather mild additional conditions, then the $\mathscr{K}$-structure space $S(X, Y, \alpha)$ is the Stone-Čech compactification of the range of $\alpha$. The results in the present paper generalize results in both [5] and [6] since by taking $X=Y$ and $\alpha$ to be the identity map we get results in the latter as special cases.

In $\S 2$, we state (without proof) revisions of results in [5] that we need in order to prove the main theorem here. Section 3 contains a brief discussion of sandwich semigroups and some of their elementary properties and it is there that the main theorem of the paper is proved. In $\S 4$, we discuss some applications of the main result and some examples.

2. Revisions of some previous results. Although we are primarily interested in $\mathscr{K}$-structure spaces, whose definition we have recalled in the introduction, there are generally many structure spaces for a semigruop, one for each left ideal. We now give the more general definition so we can state the revision of the results in [5] that we need in this paper. The proofs of these results when $T$ has no left identity are the same as in [5] or at worst are obvious modifications thereof

Definition 2.1. Let $S$ be any semigroup and $Z$ any left ideal of $S$. A nonempty subset $A$ of $S \times Z$ is called a bond if for each finite subset $\left\{\left(s_{i}, z_{i}\right)\right\}_{i=1}^{n}$ of $A$ the system of equations $\left\{s_{i} x=z_{i}\right\}_{i=1}^{n}$ has a common solution $x$ in $Z$. A maximal bond is called an ultrabond. The set of all ultrabonds is denoted by $\mathscr{U}(S, Z)$.

For any $v \in Z$, the set $A_{v}=\{(s, s v): s \in S\}$ is an ultrabond. In [5] it was assumed that $S$ has a left identity to prove this. $A_{v}$ is an ultrabond regardless of whether or not $S$ has a left identity.

Definition 2.2. An ultrabond of the form $A_{v}$ is called a princi- 
pal ultrabond and the set of all principal ultrabonds is denoted by $\mathscr{R}(S, Z)$.

We topologize $\mathscr{C}(S, Z)$ by taking the sets of the form

$$
H(s, z)=\{A \in \mathscr{U}(S, Z):(s, z) \in A\}
$$

as a subbasis for the closed subsets.

Definition 2.3. $\mathscr{U}(S, Z)$ with the topology just described is called the structure space of the pair $(S, Z)$. The subspace consisting of all the principal ultrabonds will be denoted by $\mathscr{R}(S, Z)$.

Each element $a \in S$ iduces in a natural way a mapping $\lambda_{a}$ from $\mathscr{R}(S, Z)$ into $\mathscr{R}(S, Z)$. Simply, define $\lambda_{a}\left(A_{v}\right)=A_{a v}$.

THEOREM 2.4. Let $S$ be a semigroup and let $Z$ be a left ideal of $S$. Then for each element $a$ in $S$, the function $\lambda_{a}$ is a continuous mapping from $\mathscr{R}(S, Z)$ into $\mathscr{R}(S, Z)$.

Definition 2.5. The pair $(S, Z)$ is said to be admissible if for any ultrabond $A \in \mathscr{C} H\left(s_{1}, z_{1}\right)$ ( $\mathscr{C}$ denotes complement), there exist $\left(s_{2}, z_{2}\right)$ and $\left(s_{3}, z_{3}\right)$ in $S \times Z$ such that

$$
A \in \mathscr{C} H\left(s_{2}, z_{2}\right) \subseteq H\left(s_{3}, z_{3}\right) \leqq \mathscr{C} H\left(s_{1} z_{1}\right) .
$$

The following will be crucial for the proof of our main result.

THEOREM 2.6. Suppose the pair $(S, Z)$ is admissible. Then $\mathscr{L}(S, Z)$ is a Hausdorff compactification of $\mathscr{R}(S, Z)$. Moreover, for each $a \in S$, the function $\lambda_{a}$ in $S(\mathscr{R}(S, Z))$ has a unique extension to a function $\hat{\lambda}_{a}$ in $S(\mathscr{U}(S, Z))$.

3. The $\mathscr{K}$-structure space of a sandwich semigroup. Sandwich semigroups were essentially introduced in [3]. For additional results on sandwich semigroups as well as references to other related papers, one may consult [4], [10], [11] and [13] through [15]. Actually in some of the latter papers, the domains of the functions comprising the sandwich semigroup did not necessarily need to be the entire space. In this paper, of course, they do so that what we call a sandwich semigroup here is a special case of what is studied in some of the latter papers. This section contains the main theorem of the paper and the major portion of this section is devoted to a proof of that theorem. However, before we begin that task, we verify a few of the other remarks made about sandwich semigroups in the introduction. The first is 
THEOREM 3.1. $S(X, Y, \alpha)$ has a left identity if and only if $\alpha$ maps $Y$ homeomorphically onto a retract of $X$.

Proof. Let $l$ be a left identity of $S(X, Y, \alpha)$. The function $\alpha \circ l$ is a continuous selfmap of $X$. Moreover, we see that

$$
(\alpha \circ l) \circ(\alpha \circ l)=\alpha \circ(l \circ \alpha \circ l)=\alpha \circ(l l)=\alpha \circ l
$$

so that $\alpha \circ l$ is an idempotent (with respect to composition) continuous selfmap of $X$. Thus, $\operatorname{Ran}(\alpha \circ l)$ (i.e., the range of $\alpha \circ l)$ ) is a retract of $X$. Evidently, $\operatorname{Ran}(\alpha \circ l) \cong \operatorname{Ran} \alpha$. To verify the reverse inclusion, take any $x \in \operatorname{Ran} \alpha$. Then $x=\alpha(y)$ for some $y \in Y$. Define the function $\langle y\rangle$ by $\langle y\rangle(x)=y$ for all $x \in X$ and get

$$
\begin{aligned}
(\alpha \circ l)(x) & =\alpha \circ l \circ \alpha(y)=\alpha \circ l \circ \alpha \circ\langle y\rangle(x)=\alpha \circ(l\langle y\rangle)(x) \\
& =\alpha \circ\langle y\rangle(x)=\alpha(y)=x .
\end{aligned}
$$

We draw two conclusions from this. The first is that $\operatorname{Ran}(\alpha \circ l)=$ $\operatorname{Ran} \alpha$ and the second is that $\alpha \circ l$ is the identity on $\operatorname{Ran} \alpha$.

Now choose any $x \in X$ and $y \in Y$ and we have

$$
(l \circ \alpha)(y)=l \circ \alpha \circ\langle y\rangle(x)=(l\langle y\rangle)(x)=\langle y\rangle(x)=y .
$$

That is, $l \circ \alpha$ is identity on $Y$. Thus, $\operatorname{Ran} \alpha$ is, indeed, a retract of $X$ and $\alpha$ is a homeomorphism from $Y$ onto $\operatorname{Ran} \alpha$.

Conversely suppose $\alpha$ is a homeomorphism from $Y$ onto $\operatorname{Ran} \alpha$ which is a retract of $X$. Then $\operatorname{Ran} \alpha=\operatorname{Ran} v$ where $v$ is some idempotent continuous selfmap of $X$. Define $l=\alpha^{-1} \circ v$. Then $l \in$ $S(X, Y, \alpha)$ and using the fact that $v$ is the identity on $\operatorname{Ran} \alpha$, one readily verifies that $l$ is a left identity for $S(X, Y, \alpha)$.

When we say that $S(X, Y, \alpha)$ separates points, we mean as usual that if $a$ and $b$ are distinct points of $X$ then $f(a) \neq f(b)$ for some $f \in S(X, Y, \alpha)$.

TheOREM 3.2. Suppose $S(X, Y, \alpha)$ separates points. Then $S(X, Y, \alpha)$ has a right identity if and only if $\alpha$ maps some retract of $Y$ homeomorphically onto $X$.

Proof. Let $r$ be a right identity of $S(X, Y, \alpha)$. Then $r \circ \alpha$ is a continuous selfmap of $Y$ and moreover

$$
(r \circ \alpha) \circ(r \circ \alpha)=(r \circ \alpha \circ r) \circ \alpha=(r r) \circ \alpha=r \circ \alpha .
$$

That is, $r \circ \alpha$ is idempotent. We wish to show next that $\operatorname{Ran}(r \circ \alpha)=\operatorname{Ran} r$. We immediately have $\operatorname{Ran}(r \circ \alpha) \cong \operatorname{Ran} r$. On the other hand, take any $y \in \operatorname{Ran} r$. Then $y=r(x)$ for some $x \in X$ and we have 


$$
(r \circ \alpha)(y)=(r \circ \alpha \circ r)(x)=(r r)(x)=r(x)=y .
$$

This not only tells us that $\operatorname{Ran}(r \circ \alpha)=\operatorname{Ran} r$, in which case Ran $r$ is a retract of $Y$, but it also tells us that $r \circ \alpha$ is the identity on Ran $r$. To complete the proof we need only show that $\alpha \circ r$ is the identity on $X$. So let any $x \in X$ and $f \in S(X, Y, \alpha)$ be given. Then

$$
f(\alpha \circ r(x))=(f \circ \alpha \circ r)(x)=(f r)(x)=f(x) .
$$

Since $S(X, Y, \alpha)$ separates points, it follows that $\alpha \circ r(x)=x$. Thus, $\alpha \circ r$ is the identity on $X$ which means $\alpha$ maps Ran $r$ homeomorphically onto $X$ and the proof is complete.

REMARK. One cannot simply delete the requirement that $S(X, Y, \alpha)$ separate points and hope to prove the theorem. Examples are abundant which illustrate this. Take $X$ to be any connected space, $Y$ any totally disconnected space and $\alpha$ any continuous function from $Y$ into $X$. Then $S(X, Y, \alpha)$ is a left zero semigroup which means that everything is a right identity.

Corollary 3.3. Suppose $S(X, Y, \alpha)$ separates points. Then $S(X, Y, \alpha)$ has a two-sided identity if and only if $\alpha$ is a homeomorphism from $Y$ onto $X$.

Proof. This follows immediately from the previous two theorems.

Corollary 3.4. Suppose $S(X, Y, \alpha)$ separates points and has an identity. Then $S(X, Y, \alpha), S(X)$ and $S(Y)$ are all mutually isomorphic.

Proof. According to the previous corollary, $\alpha$ is a homeomorphism from $Y$ onto $X$. The mapping which carries $f$ into $\alpha \circ f$ is an isomorphism from $S(X, Y, \alpha)$ onto $S(X)$ and the mapping which carries $f$ into $f \circ \alpha$ is an isomorphism from $S(X, Y, \alpha)$ onto $S(Y)$.

REMARK. The previous results can all be proven in considerably more generality. For example they all have analogues within the framework of $\Delta$-structures [9] and the proofs given here carry over intact. In order to present the more general results, we would have to devote considerable space to a discussion of definitions, terminology, etc. and we feel that to be not appropriate here.

Before we can state the main theorem, we need to recall some facts about $E$-compact spaces. We use the terminology and notation of [6]. Let $E$ be a Hausdorff space. A space $X$ is $E$-completely 
regular if it is homeomorphic to a subspace of some cartesian product of copies of $E$ and it is $E$-compact if it is homeomorphic to a closed subspace of such a product. An $E$-compact space $Y$ which contains $X$ as a dense subspace is called an $E$-compactification of $X$. An important fact about $E$-compactifications is that if $E$ is compact in the usual sense, then each $E$-completely regular space $X$ has a largest $E$-compactification $\beta_{E} X$ in the sense that any other $E$-compactification of $X$ is a continuous image of $\beta_{E} X$ under a map which keeps the points of $X$ fixed. This is an immediate consequence of Theorem 4.14 of [12, p. 177]. Before stating our main theorem, we recall another concept introduced in [6].

Definition 3.5. Let $E$ be Hausdorff. A space $X$ is $E$-separated if it is Hausdorff and for each pair $H$ and $K$ of disjoint closed subsets of $X$, there exists a continuous function $f$ from $X$ into $E$ and two distinct points $p$ and $q$ of $E$ such that $f(x)=p$ for $x \in H$ and $f(x)=q$ for $x \in K$.

It is clear that every $E$-separated space is normal. It is also easy to see that every closed subspace of an $E$-separated space is $E$-separated. We are now in a position to state our main result which concerns the $\mathscr{K}$-structure space of a sandwich semigroup.

MAIN THeOREM 3.6. Suppose $E$ is compact, $X$ is E-separated and $Y$ contains a copy of $E$ on which $\alpha$ is injective. Suppose also that $\alpha$ is a closed map of $Y$ into $X$ and every continuous function on $\alpha(Y)$ to $E$ can be continuously extended to a function of $X$ to $E$. Then the $\mathscr{K}$-structure space of $S(X, Y, \alpha)$ is $\beta_{E}(\alpha(Y))$, the largest E-compactification of $\alpha(y)$.

Note that $\alpha(Y)$ is $E$-completely regular, being a closed subspace of an $E$-separated space $X$ and hence it is meaningful to talk of $\beta_{E}(\alpha(Y))$.

We will prove the theorem after a series of lemmas. Let $S$ denote the semigroup $S(X, Y, \alpha)$ and $K$ its kernel. $K=\{\langle y\rangle: y \in y\}$ where $\langle y\rangle$ denotes the constant map which maps every element of $X$ into $y$. Furthermore, it is easy to show that

(1) For any $f \in S$ and $\langle y\rangle,\langle z\rangle$ in $K, f \cdot\langle y\rangle=\langle z\rangle$ if and only if $f(\alpha(y))=z$.

(2) A subset $A$ of $S \times K$ is a bond if and only if $\left\{f^{-1}(z)\right.$ : $(f,\langle z\rangle) \in A\} \cup\{\operatorname{Ran} \alpha\}$ has the finite intersection property.

(3) $A$ is an ultrabond if and only if $(f,\langle z\rangle) \notin A$ implies $f^{-1}(z) \cap$ $g_{1}^{-1}\left(y_{1}\right) \cap \cdots \cap g_{n}^{-1}\left(y_{n}\right) \cap \operatorname{Ran} \alpha=\varnothing$ for a finite subfamily $\left\{\left(g_{i},\left\langle y_{i}\right\rangle\right)\right\}_{i=1}^{n}$ of $A$. 
We also need the following lemma which is easy to prove.

Lemma 3.7. Suppose $X$ is E-separated. For any pair $H$ and $K$ of disjoint closed subsets of $X$, there exists an $f \in S$ and two distinct points $a$ and $b$ of $Y$ such that $f(x)=a$ for $x \in H$ and $f(x)=b$ for $x \in K$.

Throughout the rest of this section, $X, Y, \alpha$ are supposed to satisfy the hypothesis of the main theorem.

LEMMA 3.8. The pair $(S, K)$ is admissible.

Proof. Suppose $A$ is an ultrabond and $A \in \mathscr{C} H(f,\langle z\rangle)$. Then there exist $\left\{\left(g_{i},\left\langle y_{i}\right\rangle\right)\right\}_{i=1}^{K} \in A$ such that

$$
f^{-1}(z) \cap W=\varnothing, \quad \text { where } \quad W=\bigcap_{i=1}^{N} g_{i}^{-1}\left(y_{i}\right) \cap \operatorname{Ran} \alpha .
$$

Since $W \subseteq \mathscr{C} f^{-1}(z)$ and $X$ is normal, there exists an open set $G$ and a closed set $K$ such that $W \subseteq G \leqq K \subseteq \mathscr{C} f^{-1}(z)$. By Lemma 3.7 there exists two functions $h, k \in S$ and points $a, b, p, q$ in $Y$ such that $a \neq b$ and $p \neq q$ and

$$
\begin{array}{lll}
h(x)=a & \text { for } & x \in X-G \\
h(x)=b & \text { for } & x \in W \\
k(x)=p & \text { for } & x \in K
\end{array}
$$

and

$$
k(x)=q \text { for } \quad x \in f^{-1}(z) .
$$

Then $W \subseteq \mathscr{C} h^{-1}(a) \subseteq k^{-1}(p) \subseteq \mathscr{C} f^{-1}(z)$. To complete the proof we show that $A \in \mathscr{C} H(h,\langle a\rangle) \subseteq H(k,\langle p\rangle) \subseteq \mathscr{C} H(f,\langle z\rangle)$.

Since $W \subseteq \mathscr{C} h^{-1}(a)$ it follows that $A \in \mathscr{C} H(h,\langle a\rangle)$. Suppose now $B \in \mathscr{C} H(h,\langle a\rangle)$. Then there exist $\left\{\left(h_{i},\left\langle a_{i}\right\rangle\right)\right\}_{i=1}^{M}$ in $B$ such that

$$
h^{-1}(a) \cap \bigcap_{i=1}^{M} h_{1}^{-1}\left(a_{i}\right) \cap \operatorname{Ran} \alpha=\varnothing .
$$

If $B \notin H(k,\langle p\rangle)$ then there exist $\left\{\left(k_{i},\left\langle b_{i}\right\rangle\right)\right\}_{i=1}^{K}$ in $B$ such that

$$
k^{-1}(p) \cap \bigcap_{i=1}^{K} k_{i}^{-1}\left(b_{i}\right) \cap \operatorname{Ran} \alpha=\varnothing .
$$

Since $\mathscr{C} h^{-1}(a) \subseteq k^{-1}(p),(5)$ and (6) together imply that $\bigcap_{i=1}^{M} h_{i}^{-1}\left(a_{i}\right) \cap$ $\bigcap_{i=1}^{K} k_{i}^{-1}\left(b_{i}\right) \cap \operatorname{Ran} \alpha=\varnothing$.

This contradicts that $B$ is a bond and hence $B \in H(k,\langle p\rangle)$, proving that $\mathscr{C} H(h,\langle a\rangle) \subseteq H(k,\langle p\rangle)$.

To show that $H(k,\langle p\rangle) \subseteq \mathscr{C} H(f,\langle z\rangle)$, suppose $B \in H(k,\langle p\rangle)$ and 
$B \in H(f,\langle z\rangle)$. Then $(f,\langle z\rangle)$ and $(k,\langle p\rangle)$ both belong to $B$ but $k^{-1}(p) \cap$ $f^{-1}(z)=\varnothing$, which is a contradiction. This proves that $H(k,\langle p\rangle) \subseteq$ $\mathscr{C} H(f,\langle z\rangle)$, and the lemma is proved.

The following lemma is easy to prove.

Lemma 3.9. For $y, z \in Y, A_{\langle y\rangle}=A_{\langle z\rangle}$ if and only if $\alpha(y)=\alpha(z)$.

Let $\mathscr{U}$ denote $\mathscr{C}(S)$ and $\mathscr{R}$ denote the set $\left\{A_{\langle y\rangle}: y \in y\right\}$. By Lemma 3.8 and Theorem $2.6 \mathscr{C}$ is a Hausdorff compactification of $\mathscr{R}$. Let $e$ be the mapping of $Y$ into $\mathscr{R}$ defined by $e(y)=A_{\langle y\rangle}$.

LEMma 3.10. $e$ is continuous and closed.

Proof. Since sets of the form $H(f,\langle z\rangle) \cap \mathscr{R}$ constitute a subbase of closed subsets of $\mathscr{R}$ and $e^{-1}[H(f,\langle\boldsymbol{z}\rangle) \cap \mathscr{R}]=(f \circ \alpha)^{-1}(\boldsymbol{z})$, it follows that $e$ is continuous. Suppose now that $K$ is a closed subset of $Y$ and $A_{\langle y\rangle} \notin e[K]$. Then $\alpha(y) \notin \alpha[K]$ and $\alpha[K]$ is closed. By Lemma 3.7, there exist $f \in S$ and $a, b \in Y, a \neq b$ such that $f(\alpha(y))=a$ and $f(x)=b$ for every $x \in \alpha[K]$.

Then $\mathscr{C} H(f,\langle b\rangle)$ is a neighborhood of $A_{\langle y\rangle}$ which does not intersect $e[K]$ showing that $e[K]$ is closed.

Lemma 3.10 shows that $\mathscr{R}$ has the quotient topology induced by the map $e$ on $Y$. Since $\alpha$ is by hypothesis a continuous closed map of $Y$ it follows that $\alpha[Y]$ also has the quotient topology. Now there is a natural map $g$ of $\alpha[Y]$ onto $\mathscr{R}$ defined by $g(\alpha(y))=A_{\langle y\rangle}$.

Lemma 3.9 shows that this is well defined and injective and the preceding discussion implies that $g$ is a homeomorphism. Thus we have proved

\section{Corollary 3.11. $\alpha[Y]$ is homeomorphic to $\mathscr{R}$.}

Proof of the main theorem. We begin by proving that the structure space $\mathscr{U}$ is $E$-compact. We have already observed that $\mathscr{Q}$ is a compact Hausdorff space. Thus, if an embedding into a cartesian product of copies of $E$ exists, $\mathscr{C}$ must necessarily be embedded as a closed subset. Consequently, we need only prove the existence of an embedding. According to Theorem 2.1 of [12, p. 165], it will be sufficient to show that for each closed subset $W$ of $\mathscr{W}$ and each $A \in \mathscr{C}$ with $A \notin W$, there exists a continuous function $f$ from $\mathscr{Q}$ into $E$ and a point $q$ in $E$ such that $f(B)=q$ for $B \in W$ and $f(A) \neq q$. The proof which follows is a modification of the corresponding proof in [6].

Now $W$ is closed and $A \notin W$. Therefore there exists a finite 
subfamily $\left\{\left(g_{i},\left\langle y_{i}\right\rangle\right)\right\}_{i=1}^{N}$ of $S \times K$ such that

$$
A \notin W^{*}, W \subseteq W^{*}
$$

where $W^{*}=\bigcup_{i=1}^{N} H\left(g_{i}\left\langle y_{i}\right\rangle\right)$. Since $A \notin W^{*}$, there exist by (3), finite subfamilies $\left\{\left(h_{i j},\left\langle v_{i j}\right\rangle\right)\right\}_{i=1}^{N_{i}}$ of $A$ such that $g_{i}^{-1}\left(y_{i}\right) \cap V_{i}=\varnothing$ where

$$
V_{i}=\bigcap_{i=1}^{N_{i}} h_{i j}^{-1}\left(v_{i j}\right) \cap \operatorname{Ran} \alpha \text { for } 1 \leqq i \leqq N .
$$

Now let $V^{*}=\bigcup_{i=1}^{N} V_{i}$ and let $H=\bigcup_{i=1}^{N} g_{i}^{-1}\left(y_{i}\right)$. Then $V^{*}$ and $H$ are two disjoint closed subsets of $X$ and hence there exist a continuous function $s$ mapping $X$ into $E^{*}$ and two distinct points $p$ and $q$ of $E^{*}$ such that

$$
s(x)=p \text { for } x \in V^{*} \text { and } s(x)=q \text { for } x \in H \text {, where } E^{*} \text { is } h(E),
$$

(8) $h$ being a homeomorphism of $E$ into $Y$ which results from the hypothesis.

Now the mapping $\lambda_{s}: \mathscr{R} \rightarrow \mathscr{R}$ defined by $\lambda_{s}\left(A_{\langle y\rangle}\right)=A_{\langle s(\alpha(y))\rangle}$ is continuous by Theorem 2.4 and has a unique extension to a continuous selfmap $\hat{\lambda}_{s}$ of $\mathscr{C}$ by Lemma 3.8 and Theorem 2.6. Now we assert that

$$
\widehat{\lambda}_{s}(\mathscr{U}) \cong e\left[E^{*}\right]
$$

and

$$
\widehat{\lambda}_{s}(A)=e(p) \text { and } \hat{\lambda}_{s}(B)=e(q) \text { for } B \in W^{*} .
$$

Now $s(X) \subseteq E^{*}$. Therefore $\hat{\lambda}_{s}\left(A_{\langle y\rangle}\right)=A_{\langle s(\alpha(y))\rangle} \in e\left[E^{*}\right]$ for every $y \in Y$. Since $\mathscr{R}$ is dense in $\mathscr{C}$ and $E^{*}$ is compact, we have

$$
\hat{\lambda}_{s}(\mathscr{C})=\hat{\lambda}_{s}(\operatorname{cl} \mathscr{R}) \leqq \operatorname{cl}\left(\hat{\lambda}_{s}(\mathscr{R})\right) \leqq \operatorname{cl} e\left[E^{*}\right]=e\left[E^{*}\right],
$$

where cl denotes the closure. This verifies (9). Now we shall show that

$$
A \in \operatorname{cl}\left\{A_{\langle y\rangle}: \alpha(y) \in V^{*}\right\} .
$$

Let $\mathscr{C}\left[\bigcup_{i=1}^{M} H\left(k_{i},\left\langle r_{i}\right\rangle\right)\right]$ be a basic neighborhood of $A$. Then by (3), there exist finite subfamilies $\left\{\left(t_{i j},\left\langle a_{i j}\right\rangle\right)\right\}_{j=1}^{M_{i}}$ of $A$ such that $k_{i}^{-1}\left(r_{i}\right) \cap$ $U_{i}=\varnothing$ where $U_{i}=\bigcap_{j=1}^{M i} t_{i j}^{-1}\left(a_{i j}\right) \cap \operatorname{Ran} \alpha$.

Now there exists $y$ in $Y$ such that $\alpha(y) \in V^{*} \cap U_{1} \cap \cdots \cap U_{M}$. But, then $\alpha(y) \notin k_{i}^{-1}\left(r_{i}\right), i=1,2, \cdots, M$ so that $\left(k_{i},\left\langle r_{i}\right\rangle\right) \notin A_{\langle y\rangle}$. Therefore, $A_{\langle y\rangle} \in \mathscr{C}\left[\bigcup_{i=1}^{M} H\left(k_{i},\left\langle r_{i}\right\rangle\right)\right]$, and this proves (11). Now for $\alpha(y) \in$ $V^{*}, s(\alpha(y))=p$ and we have $\hat{\lambda}_{s}\left(A_{\langle y\rangle}\right)=A_{\langle s\{\alpha(y)\rangle\rangle}=e(p)$.

This, together with (11) implies that $\hat{\lambda}_{s}(A)=e(p)$, which is the first half of (10). In the same way as we verified (11), we can show that if $B \in W^{*}$ then $B \in \operatorname{cl}\left\{A_{\langle y\rangle}: \alpha(y) \in H\right\}$ and since $s(x)=q$ 
for $x \in H$, it follows that $\hat{\lambda}_{s}\left(A_{\langle y\rangle}\right)=e(q)$ for every $y$ such that $\alpha(y) \epsilon$ $H$. Therefore $\hat{\lambda}_{s}(B)=e(q)$ for each $B \in W^{*}$ and this completes the proof of the statement (10). Now, $e$ is injective on $E^{*}$ and $\hat{\lambda}_{s}(\mathscr{U}) \subseteq$ $e\left[E^{*}\right]$. Therefore, $(e \circ h)^{-1} \circ \hat{\lambda}_{s}=f$ is a continuous function from $\mathscr{U}$ to $E$ and we have a point $a\left(=h^{-1}(q)\right)$ in $E$ such that $f(B)=a$ for every $B \in W$ and $f(A) \neq a$, which was to be shown in order to conclude that $\mathscr{Q}$ is $E$-compact.

Now we are in a position to show that $\mathscr{C}$ is $\beta_{E}(\alpha(y))$. Actually, we show that $\mathscr{C}$ is $\beta_{E}(\mathscr{R})$ but since the canonical map $g$ (described after Lemma 3.10) maps $\alpha(E)$ homeomorphically onto $\mathscr{R}$ we identify the two spaces. In order to conclude that $\mathscr{C}$ is $\beta_{E}(\mathscr{R})$ it is sufficient, according to Theorem 4.14 of $[12$, p. 177] to show that $\mathscr{C}$ is $E$-compact and that every continuous function from $\mathscr{R}$ to $E$ can be continuously extended to a function which maps $\mathscr{C}$ into $E$. We have yet to verify the latter and this is what we shall do now. Let $f$ be a continuous function from $\mathscr{R}$ to $E$. Then $f \circ g$ is a continuous function on $\alpha(Y)$ to $E$. Let $(f \circ g)^{*}$ denote an extension of $f \circ g$ to a continuous function on $X$ to $E$. Now $h \circ(f \circ g)=k$ belongs to $S$ and

$$
\lambda_{k}\left(A_{\langle y\rangle}\right)=A_{\langle k\langle\alpha(y)\rangle\rangle}=A_{\langle h \circ f \circ g\langle\alpha(y)\rangle\rangle}=A_{\langle h \circ f\langle A\langle y\rangle\rangle\rangle}=e \circ h \circ f\left(A_{\langle y\rangle}\right) .
$$

Therefore, $\lambda_{k}=e \circ h \circ f$. Now $\lambda_{k}$ has a unique continuous extension $\hat{\lambda}_{k}$ to a selfmap of $\mathscr{U}$. We then have $\hat{\lambda}_{k}(\mathscr{C})=\hat{\lambda}_{k}(\operatorname{cl} \mathscr{R}) \subseteq$ $\operatorname{cl}\left(\hat{\lambda}_{k}(\mathscr{R})\right)=\operatorname{cl}(e \circ h \circ f(\mathscr{R})) \leqq \operatorname{cl}(e \circ h(E))=e \circ h(E)$.

The last equality is obtained because $E$ is compact and $e \circ h$ is a homeomorphism on $E$. The function $(e \circ h)^{-1} \circ \hat{\lambda}_{k}$ is the desired extension of $f$ because $(e \circ h)^{-1} \circ \hat{\lambda}_{k}\left(A_{\langle y\rangle}\right)=(e \circ h)^{-1} \circ \lambda_{k}\left(A_{\langle y\rangle}\right)=(e \circ h)^{-1} \circ$ $e \circ h \circ f\left(A_{\langle y\rangle}\right)=f\left(A_{\langle y\rangle}\right)$.

This completes the proof of the main theorem. If, in the main theorem, we take $E$ to be the closed unit interval, we immediately get

Corollary 3.12. Suppose $X$ is normal and Hausdorff, $\alpha$ a continuous closed map of $Y$ into $X$ and $Y$ contains an arc on which $\alpha$ is injective. Then the $\mathscr{K}$-structure space of $S(X, Y, \alpha)$ is the Stone-Čech compactification of $\alpha(Y)$.

Corollary 3.13. Let $X$ be a normal 0-dimensional Hausdorff space and let $\alpha$ be a continuous closed map of $Y$ into $X$. Then the $\mathscr{K}$-structure space of $S(X, Y, \alpha)$ is the Stone-Čech compactification of $\alpha(Y)$.

Proof. Here again we apply the main theorem and in this case we take $E$ to be the two point discrete space $\mathscr{D}$. First we assume 
that $\alpha$ is not constant, then $Y$ contains a copy of $\mathscr{D}$ on which $\alpha$ is injective. One can show that $X$ is $\mathscr{D}$-separated exactly as it is done in the proof of Corollary 2 of [6]. Now we show that every continuous function $f$ on $\alpha(Y)$ to $\mathscr{D}$ can be continuously extended to $X$. Let $p$ and $q$ be the distinct points of $\mathscr{D}$. Then $f^{-1}(p)$ and $f^{-1}(q)$ are disjoint closed subsets of $\alpha(Y)$ and consequently that of $X$ as $\alpha(Y)$ is closed in $X$. Since $X$ is $\mathscr{D}$-separated there exists a continuous function $\hat{f}$ on $X$ to $\mathscr{D}$ such that $\hat{f}(x)=p$ for $x \in f^{-1}(p)$ and $\hat{f}(x)=q$ for $x \in f^{-1}(q)$. This $\hat{f}$ is the desired extension. Now our main theorem implies that the $\mathscr{K}$-structure space of $S(X, Y, \alpha)$ is $\beta_{\mathscr{D}}(\alpha(y))$, the largest $\mathscr{D}$-compactification of $\alpha(y)$. Now one proves exactly as in the proof of Corollary 2 of [6] that $\beta(\alpha(y)$ is a $\mathscr{D}$ compactification of $\alpha(y)$ which in turn implies that $\beta_{\mathscr{D}}(\alpha(Y))=$ $\beta(\alpha(Y))$ and the $\mathscr{K}$-structure space of $S(X, Y, \alpha)$ is $\beta(\alpha(Y))$.

Suppose now that $\alpha$ is a constant map of $Y$ into $X$, say $\alpha(y)=$ $x_{0}$ for every $y \in Y$. Let $A$ be an ultrabond for the pair $(S, K)$ where $K$ is the kernel of $S(X, Y, \alpha)$. Suppose $(f,\langle y\rangle) \in A$. Then by definition there exists $y^{\prime} \in Y$ such that $f \cdot\left\langle y^{\prime}\right\rangle=\langle y\rangle$. But $f \cdot\left\langle y^{\prime}\right\rangle=$ $\left\langle f\left(\alpha\left(y^{\prime}\right)\right)\right\rangle=\left\langle f\left(x_{0}\right)\right\rangle$. Thus $A \subseteq\left\{\left(f,\left\langle f\left(x_{0}\right)\right\rangle\right): f \in S(X, Y, \alpha)\right\}$ which itself is an ultrabond. Therefore $A=\left\{\left(f,\left\langle f\left(x_{0}\right)\right\rangle\right): f \in S\right\}$ and $\mathscr{C}(S)$ consists of just one point and consequently is the Stone-Čech compactification of $\alpha(Y)$.

4. Examples, applications and concluding remarks. If one takes $X=Y$ and $\alpha$ to be the identity map, then $S(X, Y, \alpha)$ is just $S(X)$ the semigroup, under composition, of all continuous selfmaps of $X$. It follows immediately from the results of the previous section that for a number of spaces $X$, the $\mathscr{K}$-structure space of $S(X)$ is $\beta X$, the Stone-Čech compactification of $X$. This generalizes results in both [5] and [6].

For further examples, take $X$ to be any normal space, choose any closed subspace $Y$ which contains an arc and denote by $S(X, Y)$ the subsemigroup of $S(X)$ consisting of all functions which map $X$ into $Y$. Then $S(X, Y)$ is really a sandwich semigroup (the sandwich map is the identity map on $Y$ ) which satisfies the hypothesis of the main theorem and it follows that its $\mathscr{K}$-structure space is $\beta Y$. For a specific example of this type, take $X$ to be the space of real numbers $\boldsymbol{R}$ and take $Y$ to be union of the unit interval with the subspace $N$ of natural numbers. Then the $\mathscr{K}$-structure space of $S(R, Y)$ is the free union of $\beta N$ with an arc.

For any closed continuous function $\alpha$ from $\boldsymbol{R}$ into $\boldsymbol{R}$ which is injective on some nondegenerate subinterval, there are precisely three possibilities for the $\mathscr{K}$-structure space of $S(\boldsymbol{R}, \boldsymbol{R}, \alpha)$. The space $\mathscr{U}$ is either an arc, $\beta \boldsymbol{R}$ or $\beta \boldsymbol{R}^{+}$where $\boldsymbol{R}^{+}$is the space of 
nonnegative real numbers. One easily verifies that any continuous selfmap of any Euclidean $n$-space which carries unbounded sets into unbounded sets, is also a closed map. Thus all polynomial functions $P$ from $\boldsymbol{R}$ into $\boldsymbol{R}$ are closed and any polynomial function $Q$ from $\boldsymbol{C}$ into $C$ is also closed where $C$ denotes the field of complex numbers. Of course, $Q$ must actually map $C$ onto $C$ since $C$ is algebraically closed. There are only two possibilities for the $\mathscr{K}$-structure space $\mathscr{U}$ of $S(\boldsymbol{R}, \boldsymbol{R}, P)$. The space $\mathscr{C}$ is $\beta \boldsymbol{R}$ if and only if $P$ is of odd degree and it is $\beta \boldsymbol{R}^{+}$if and only if $P$ is of even degree. The $\mathscr{K}$ structure space of $S(C, C, Q)$ is always $\beta \boldsymbol{R}^{2}$ regardless of the degree of $Q$. All these assertions follow immediately from the theorem.

Now let $S$ and $T$ be any two semigroups with kernels. If $S$ and $T$ are isomorphic then certainly the two $\mathscr{K}$-structure spaces $\mathscr{U}(S)$ and $\mathscr{U}(T)$ are homeomorphic. The converse, of course, is far from being true. For example if $G$ is any group, then $\mathscr{U}(G)$ has the cofinite topology [8, p. 266]. The cardinality of $\mathscr{C}(G)$ agrees with that of $G$ so if $G$ and $H$ are any two groups of the same order (finite or infinite) then $\mathscr{U}(G)$ and $\mathscr{U}(H)$ will be homeomorphic. For another type of example, $S(\boldsymbol{R})$ and $S(\beta \boldsymbol{R})$ are not isomorphic [8, p. 256] but $\mathscr{\mathscr { C }}(S(\boldsymbol{R}))=\mathscr{U}(S(\beta \boldsymbol{R}))=\beta \boldsymbol{R}$, the StoneČech compactification of the reals.

Nevertheless, with certain restrictions on $X$ and $Y$, the existence of a homeomorphism between $\mathscr{U}(S(X))$ and $\mathscr{C}(S(Y))$ will imply the existence of an isomorphism between $S(X)$ and $S(Y)$. This is included in the following

TheOREM 4.1. Suppose that both $X$ and $Y$ are first countable normal spaces and suppose that each of them either contains an arc or is O-dimensional. Then the following statements are equivalent

4.1.1 The two $\mathscr{K}$-structure spaces $\mathscr{U}(S(X))$ and $\mathscr{U}(S(Y))$ are homeomorphic.

4.1.2 The two semigroups $S(X)$ and $S(Y)$ are isomorphic.

4.1.3 The spaces $X$ and $Y$ are homeomorphic.

Proof. Suppose 4.1.1 holds. Then by the results of the previous section there is a homeomorphism $h$ from $\beta X$ onto $\beta Y$. No point in $\beta X-X$ is a $G_{\delta}$ in $\beta X$ while each point in $X$ is a $G_{\delta}$ in $\beta X$, in fact it has a countable base in $\beta X[1$, p. 132]. Of course, the same thing is true of $Y$ and $\beta Y$ so that the homeomorphism $h$ must carry $X$ onto $Y$ and we see that 4.1 .1 implies 4.1.3. It is immediate that 4.1.3 implies 4.1.2 and that 4.1.2 implies 4.1.1.

We conclude the paper with one more result of this type. Recall that a subspace $Y$ is tamely embedded in a space $X$ if every 
homeomorphism from $Y$ to some copy of $Y$ in $X$ can be extended to a homeomorphism mapping $X$ onto $X$.

THEOREM 4.2. Let $X$ be normal space and let $Y$ and $Z$ be two first countable closed subspaces which are tamely embedded in $X$. Suppose further that $X$ is either O-dimensional or that both $Y$ and $Z$ contain arcs. Let $S(X, Y)$ and $S(X, Z)$ denote the semigroups, under composition of all continuous functions mapping $X$ into $Y$ and $X$ into $Z$ respectively. Then the following statements are equivalent.

4.2.1 The two K्र-structure spaces $\mathscr{U}(S(X, Y))$ and $\mathscr{U}(S(X, Z))$ are homeomorphic.

4.2.2 The two semigroups $S(X, Y)$ and $S(X, Z)$ are isomorphic.

4.2.3 The two subspaces $Y$ and $Z$ are homeomorphic.

Proof. Suppose 4.2.1 holds. By results in the previous section $\beta Y$ is homeomorphic to $\beta Z$ and just as in the proof of the previous theorem, this implies that $Y$ and $Z$ are homeomorphic. Thus 4.2.1 implies 4.2.3. If $Y$ and $Z$ are homeomorphic, then there exists a homeomorphism $h$ from $X$ onto $X$ carrying $Y$ onto $Z$ since the latter are tamely embedded in $X$. The mapping which takes $f$ into $h \circ f \circ h^{-1}$ is an isomorphism from $S(X, Y)$ onto $S(X, Z)$ so that 4.2.3 implies 4.2.2. It is evident that 4.2.2 implies 4.2 .1 and the proof is complete.

ACKNowledgment. This paper was written while the first and third authors were visiting Universidade Federal de Goiás. They wish to express their appreciation to the University for its financial support.

\section{REFERENCES}

1. L. Gillman and M. Jerison, Rings of Continuous Functions, Springer-Verlag, 1976.

2. H. O. Kim, On the product of structure spaces, Kyungpook Math. J., 11 (1971), 29-32.

3. K. D. Magill, Semigroup structures for families of functions, I, J. Aust. Math. Soc., 7 (1967), 81-94.

4. - Semigroup structures for families of functions, II, J. Aust. Math. Soc., 7 (1967), 95-107.

5. - Topological spaces determined by left ideals of semigroups, Pacific Math. J., (2) 24 (1968), 319-330.

6. - Structure spaces of semigroups of continuous functions, Trans. Amer. Math. Soc., 149 (1970), 595-600.

7. —_ $\mathscr{K}$-Structure spaces of semigroups generated by idempotents, London Math. J., (2) 3 (1971), 321-325.

8. - A survey of semigroups of continuous self maps, Semigroup Forum, 11 (1975), 189-282. 
9. K. D. Magill and S. Subbiah, Green's relations for regular elements of semigroups of endomorphisms, Canad. J. Math., 26 (1974), 1484-1497.

10. - Green's relations for regular elements of Sandwich semigroups, I, general results, Proc. London Math. Soc., 3 (31), 194-210.

11. - Green's relations for regular elements of Sandwich semigroups, II; semigroups of continuous functions, J. Aust. Math. Soc., 25 (1978), 45-65.

12. S. Mrówka, Further results on E-compact spaces, I, Acta Math., 120 (1968), 161-185.

13. R. P. Sullivan, Automorphisms of transformation semigroups; J. Aust. Math. Soc., 20 (1975) 1, 77-84.

14. J. S. V. Symons, On a generalization of the transformation semigroup, J. Aust. Math. Soc., 19 (1975), 47-61.

15. - Embedding a semigroup of transformations, J. Aust. Math. Soc., 20 (1975), 222-224.

Received November 7, 1980.

SUNY AT BUFFALO

Buffalo, N. Y. 14215. U.S.A.

IMECC CAIXa POSTAL-1170

UNICAMP, 13100-CAMPINAS-SP

BRASIL

AND

I.I.T., KANPUR

KANPUR-208016. INDIA 


\title{
PACIFIC JOURNAL OF MATHEMATICS
}

\section{EDITORS}

\author{
DONALD BABBITT (Managing Editor) \\ University of California \\ Los Angeles, CA 90024 \\ Hugo RossI \\ University of Utah \\ Salt Lake City, UT 84112 \\ C. C. MOORE and Arthur Agus \\ University of California \\ Berkeley, CA 94720
}

\section{J. DugundjI}

Department of Mathematics

University of Southern California

Los Angeles, CA 90007

R. FinN and J. Milgram

Stanford University

Stanford, CA 94305

\section{ASSOCIATE EDITORS}
R. ARENS
E. F. BeCKENBACH
B. H. NeumanN
F. WOLF
K. YoshidA

\section{SUPPORTING INSTITUTIONS}

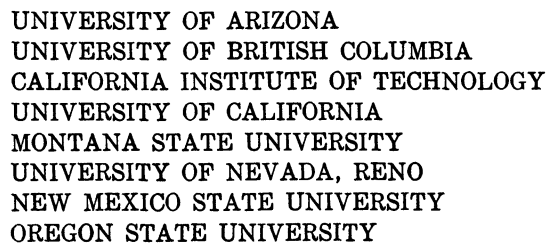

UNIVERSITY OF ARIZONA

UNIVERSITY OF BRITISH COLUMBIA

CALIFORNIA INSTITUTE OF TECHNOLOGY

UNIVERSITY OF CALIFORNIA

MONTANA STATE UNIVERSITY

UNIVERSITY OF NEVADA, RENO

NEW MEXICO STATE UNIVERSITY

OREGON STATE UNIVERSITY

\author{
UNIVERSITY OF OREGON \\ UNIVERSITY OF SOUTHERN CALIFORNIA \\ STANFORD UNIVERSITY \\ UNIVERSITY OF HAWAII \\ UNIVERSITY OF TOKYO \\ UNIVERSITY OF UTAH \\ WASHINGTON STATE UNIVERSITY \\ UNIVERSITY OF WASHINGTON
}

The Supporting Institutions listed above contribute to the cost of publication of this Journal, but they are not owners or publishers and have no responsibility for its content or policies.

Mathematical papers intended for publication in the Pacific Journal of Mathematics should be in typed form or offset-reproduced, (not dittoed), double spaced with large margins. Please do not use built up fractions in the text of the manuscript. However, you may use them in the displayed equations. Underline Greek letters in red, German in green, and script in blue. The first paragraph or two must be capable of being used separately as a synopsis of the entire paper. Please propose a heading for the odd numbered pages of less than 35 characters. Manuscripts, in triplicate, may be sent to any one of the editors. Please classify according to the scheme of Math. Reviews, Index to Vol. 39. Supply name and address of author to whom proofs should be sent. All other communications should be addressed to the managing editor, or Elaine Barth, University of California, Los Angeles, California, 90024.

50 reprints to each author are provided free for each article, only if page charges have been substantially paid. Additional copies may be obtained at cost in multiples of 50 .

The Pacific Journal of Mathematics is issued monthly as of January 1966. Regular subscription rate: $\$ 102.00$ a year (6 Vols., 12 issues). Special rate: $\$ 51.00$ a year to individual members of supporting institutions.

Subscriptions, orders for numbers issued in the last three calendar years, and changes of address shoud be sent to Pacific Journal of Mathematics, P.O. Box 969, Carmel Valley, CA 93924, U.S.A. Old back numbers obtainable from Kraus Periodicals Co., Route 100, Millwood, NY 10546.

\section{PUBLISHED BY PACIFIC JOURNAL OF MATHEMATICS, A NON-PROFIT CORPORATION}

Printed at Kokusai Bunken Insatsusha (International Academic Printing Co., Ltd.). 8-8, 3-chome, Takadanobaba, Shinjuku-ku, Tokyo 160, Japan. 


\section{Pacific Journal of Mathematics}

Vol. 99, No. $2 \quad$ June, 1982

Thomas E. Armstrong and Karel Libor Prikry, On the semimetric on a

Boolean algebra induced by a finitely additive probability measure . . . .249

Walter Russell Bloom, Strict local inclusion results between spaces of

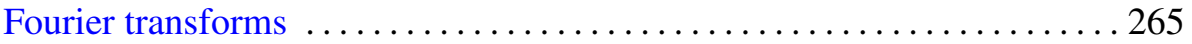

Richard Clark Brown, Notes on generalized boundary value problems in

Banach spaces. II. Infinite-dimensional extension theory ........... 271

Sui Sun Cheng, Isoperimetric eigenvalue problem of even order differential

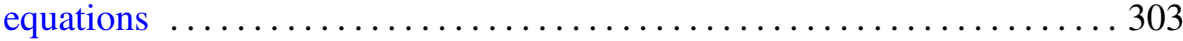

Lung O. Chung and Jiang Luh, Derivations of higher order and

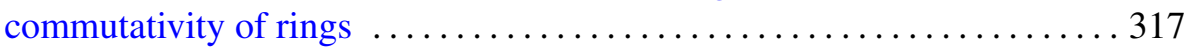

Ali Ahmad Fora, A fixed point theorem for product spaces . . . . . . . . . 327

Barry J. Gardner, Radical classes of regular rings with Artinian primitive

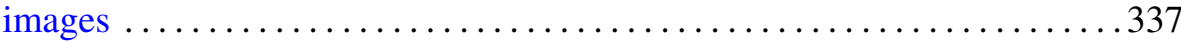

John Brady Garnett and Peter Wilcox Jones, BMO from dyadic BMO . . . 351

Allen E. Hatcher, On the boundary curves of incompressible surfaces . . . . 373

Richard Howard Hudson and Kenneth S. Williams, Resolution of

ambiguities in the evaluation of cubic and quartic Jacobsthal sums .....379

Viktor Losert, Counter-examples to some conjectures about doubly

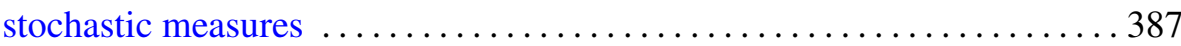

Kenneth Derwood Magill, Jr., P. R. Misra and Udai Bhan Tewari,

Structure spaces for sandwich semigroups

Mark Mandelker, Continuity of monotone functions

Kenneth Guy Miller, An index theorem and hypoellipticity on nilpotent Lie groups ......................................... 419

Evelyn M. Nelson, Homomorphisms of mono-unary algebras . . . . . . . . . 427

Marvin E. Ortel, The support of an extremal dilatation . . .

R. S. Pathak and O. P. Singh, Finite Hankel transforms of distributions . . . 439

Richard Cole Penney, The theory of ad-associative Lie algebras

Linda Ruth Sons, Zero distribution of functions with slow or moderate

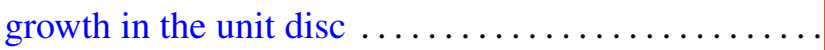

Russell Bruce Walker, Transversals to laminations 